\title{
Research on Green Modular Design
}

\author{
Lu, Zhongmei ${ }^{1}$, Huang, Yu-Che ${ }^{2, *}$, Bangjun, Cui ${ }^{1}$, and Hsiao, Ko-Jou ${ }^{3}$ \\ ${ }^{1}$ GuiZhou Vocational Technology College of Electronics \& Information, Kaili, China \\ ${ }^{2}$ Department of Industrial Design, Chaoyang University of Technology, Taichung City, Taiwan \\ ${ }^{3}$ Department of Visual Communication Design, Chaoyang University of Technology, Taichung City, Taiwan
}

\begin{abstract}
The so-called "green" product is a relative concept. Because artificial products will more or less consume resources and energy and affect the environment. If a product can be thought of in the "earth way" at the beginning of design, including products and services, it has a lower impact on the environment than the traditional method in the past to achieve the goal of sustainable use. Be green products and the modular design in the green product design method, in addition to extending the life of parts, if its main parts can have a longer life, other components can be modularized according to their average life. Make the parts in each module have the same life, make the best use of them when replacing, and can replace and repair some parts after damage, reduce waste of resources and cost, and achieve the purpose of green design. This study only discusses the modular design for the environment, and discusses the feasible design schemes of its modular design from the green assembly and disassembly methods in its modular design, and then builds the green modular design assembly with case studies and disassembly design principles, and a set of green design assembly and disassembly evaluation benchmarks. According to this method, the best green modularization feasible design scheme of this case is designed.
\end{abstract}

\section{Introduction}

In the evaluation and discussion of traditional product design procedures, as shown in Table 1, throughout the development stage of traditional product design procedures, no special consideration was given to silkcolored assembly design schemes. Generally, product assembly issues are After the design is finalized, it is jointly solved by the production and manufacturing departments. For a long time, such a product design method not only isolated the communication channels of design and manufacturing, but its designers also failed to accumulate some assembly of green design solutions. Knowledge and experience, therefore, how to use the existing product design technology concepts to analyze and evaluate the green design of the resulting design solution to assist the development of green assembly auxiliary programs is really a topic worth exploring [1-3]. Electric bicycles are an emerging product of current bicycles, and the traditional bicycle industry has also been looking forward to replacing some of the short- and medium-range motor vehicles with the green design products with environmental protection in the future in the 21 st century, so that the period can be greatly reduced. Air and noise pollution. "But there is no common design basis for the current design of electric assisted bicycles " Most of them are still on the structure of traditional bicycles plus their energy storage, drive, transmission and control systems. A reliable design basis "easy to make electric bicycles have several shortcomings of safety and comfort" and cause uncomfortable feelings for riders. It also forms many wrong design methods that violate green design and increase unnecessary waste of resources [4-6].

The combination of electric bicycles with modular applications can increase the added value of goods and market competitiveness. On the one hand, it can increase the effectiveness of leisure sports, on the other hand, it can also provide a new short-distance transportation tool for the disabled people with silver hair. From the current market survey information, we can see that the global demand for electric bicycles has grown from 300,000 to 400,000 units in 1996, and is growing and expanding at a rate of $10 \%-15 \%$ per year. For women or women, when they face uphill or upwind, they will put a considerable burden on their physical strength, which may reduce their willingness to ride. Electric bicycles modify the bicycle frame to provide its auxiliary power by adding batteries and motors. Users can ride the bicycle more easily. Even when the battery power is low, they can still rely on human power to continue their unfinished work. Distance. This study will provide an objective design and assembly process for the electric bicycle modular design research and discussion, and provide the design department of the electric vehicle industry with the evaluation criteria for assembly and disassembly. Product design reference. And it tries to make the integrated design of electric vehicles based on the modular design concept of green design related to mechanism design, and finds an ideal and appropriate design process to provide a reference for engineers to design.

*Corresponding author: che59965996@gmail.com 


\section{Literature review}

The limitations of modular design are as follows:

1.Bottom-up and top-down design method design limitations the main limitation of this method is that it emphasizes some components (trees) more than the final product (forest). Focusing on individual work and rarely considering the overall design, its efficiency will be suppressed by the efficiency of some parts. In a topdown design approach, the design team discusses the design at the top level, focusing on the characteristics of the overall product. The focus here is to complete the final product. The design process includes the establishment of scope management that aligns the desired results and the design of the parts required by the previously established scope. Therefore, the hypothetical limitation on this method is that it may cause incompatibility when designing a part of the part and meeting the required range of values [7-9].

2. Integrate top-down and bottom-up approaches. In some cases, many design processes use an integrated top-down and bottom-up approach to complete the optimized final product. In the iterative process, the design team considers the requirements from the perspective of the overall product and each individual part. In these cases, designs are usually constructed using a top-down approach, followed by an effective mix of top-down or bottom-up approaches to product design. In this way, a subassembly can be assigned to one or more designers using the best method to create individual subassemblies and parts at a previously controlled size. However, there is no special consideration and explanation for assembly and disassembly, so how to introduce production assembly and disassembly in the early stage of design is a worthwhile research direction $[10,11]$.

The basic principles of quantitative evaluation of ease of assembly were proposed by Boothroyd and Dewhurst in 1991, and the method is as follows; the disassembly and assembly actions are for the workers to decide the next action. Succession determines the continuity of action. Termination has been decided to complete. The test begins at the moment when a hand or body part begins to feel or observe an object. Succession determines the quality of an object. Terminate at the completion of the test or observation of the hand or body part. There are two main approaches to the current product assembly evaluation assistance tool, as described above, and in the "potentially removable component analysis" section, because there is only a qualitative analysis method, The basis is to rely on users to directly input data, so the feasibility of "potentially removable component analysis" automation is not high. As for assembly action analysis, there are qualitative analysis and quantitative analysis. The basis for quantitative analysis is usually time-based, that is, the time required for assembly to reflect its assembly efficiency [12-14].

Product assembly analysis (Product Design Merit, PDM). Its assembly action analysis is to decompose the assembly action into three sub-actions, namely the feeding action of the component, the fixing action of the component, and the insertion action of the component. The time required for each sub-action to evaluate the efficiency of this assembly.

The assembly action analysis of Boothro@ d \& Dewhurst company decomposes the assembly action into two sub-actions, that is, a processing action and an insertion action. The application action analysis includes component grab analysis and component alignment analysis; and the insertion action includes component positioning analysis and component insertion analysis. Boothroyd convex Dewhurst explores the characteristics of assembly sub-actions. These characteristics are divided into several levels according to the ease of assembly. Different levels of different characteristics will affect the length of time required for the assembly operation. When an assembly action is to be analyzed, the way is to compare the various relevant characteristics of each assembly sub-action separately. However, the principle of simplified components and modular design have some relevance $[15,16]$.

Of course, the product can also be designed and separated according to the disassembly order of toxic substances to avoid environmental pollution caused by heavy metals, reduce the complexity of product design, and simplify the structure as much as possible to achieve the function. For a modular design, the modules should be directly connected and have a hierarchical structure. The number of parts is reduced through functional integration, especially the number and types of screws. Therefore, the ease of assembly should also be explored. Influence to make it fully effective $[17,18]$.

\section{Research methods}

This research theory is based on the awakening of the green consciousness above, and learns that a good product must not only have traditional design concepts, but also include the overall product life cycle assessment and environmental protection considerations. However, because the overall product life cycle assessment is too extensive, this study only explores the modular design designed for the environment, and uses the green assembly and disassembly methods to combine case studies to establish green modular design guidelines. Set of green design assembly and disassembly evaluation benchmarks. And according to this method, the best green feasible design scheme of this case was improved and designed. In this study, the mechanical parts and components are listed as the main research items. Because using these mechanical parts and components, the designer enjoys greater autonomy, but for example, its technical components such as reduction gears, motors, and batteries are often limited by external technologies because they are outsourced products, and their technical fields are also different from industrial designers. Therefore, designers must not make too many choices. Design considerations for a whole rather than a single component. 


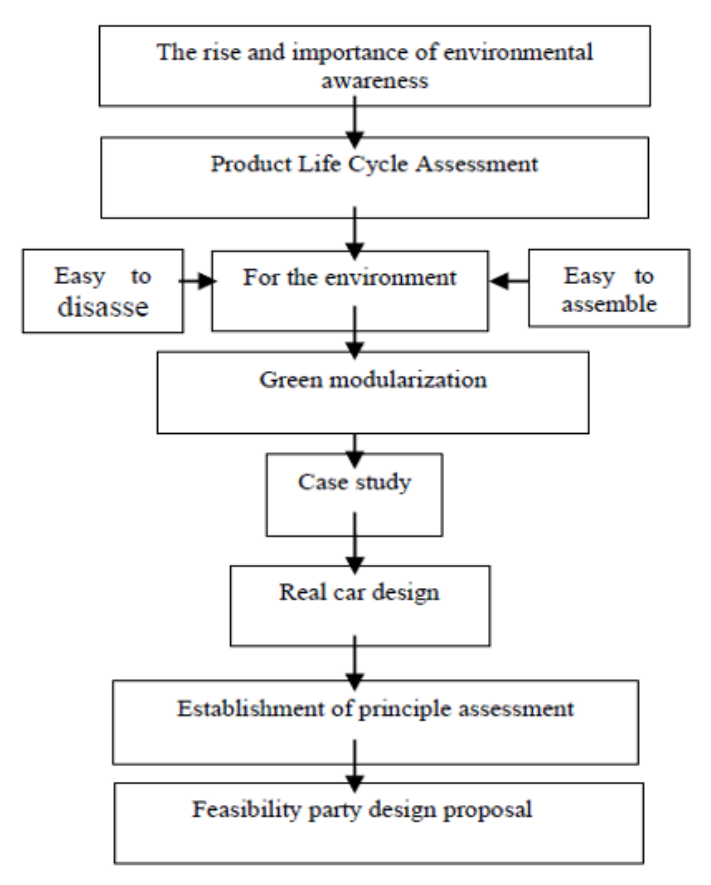

Fig. 1. Study the theoretical framework diagram.

\section{Case studies}

\subsection{Research object}

In this study, because the researcher's own work contacts and experience, he chose to focus on the case study method: the case study was taken from an electric vehicle manufacturer, and the case company designed and produced vehicle-related products. For some time, due to internationally renowned companies, they have gradually attached importance to environmental design. Based on case study method. The green assembly part will be designed by the company and its related cooperating vendors' field factory production lines and design engineers in the design department. The dismantling part has no professional recycling and dismantling personnel, so the company and its related cooperating partners will still be The on-site maintenance unit is the research object, because the field work site tends to focus on problem solving, so the research and investigation should be carried out with priority to the solution of the problem. Due to the long time it takes, try to investigate the production work orders of actual site assembly and disassembly as much as possible, and supplement the case company's abnormal assembly problem report to conduct research. The industry profile and product description of the case company are provided in the case study. Will be introduced.

\subsection{Product details}

The components of its electric bicycle are based on its main components such as its frame, drive system, energy storage system, reducer, and body shell.

\subsection{Assembly function analysis}

The functional evaluation of the green assembly design can improve the assembly efficiency of the product by improving the operation of the assembly and removing the redundant components. There are three characteristics:

(1) Review the design for unnecessary components. (2) Evaluate the assembly efficiency of the design. (3) Predict the assembly time required for the design plan when it is put into the production line in the future.

\section{Research results and literature evidence}

The assembly and disassembly design of electric bicycles in accordance with the green modular design should include:

\subsection{Design and combination of electric bicycle body shell}

(1) The combination of gluing methods does not comply with environmental protection design. ((2) Pay attention to secondary processing and environmental pollution. (3) Recyclable plastic materials should be designed.

\subsection{In terms of screw assembly design}

(1) Pay attention to the size of its assembly space and affect the lock screws. (2) When one screw is used to lock three items at the same time, pay attention to tolerances. And its assembly uses the vertical direction, the number of screws should not be too much, and mainly the same type of screws.

\subsection{The ideal assembly method that meets the assembly and manufacturing standards of green design modules should include}

(1). Modular assembly. (2). Vertical assembly. (3). Reduce the way the screws are fastened. (4). No special tools are required. (5). Reduction of parts.

\subsection{For the appearance conforming to the green module assembly design, it should include}

(1). Easy to assemble design should not affect appearance. (2). To reduce mold related problems in the future, early planning and design are required.

\subsection{The structure that complies with the green module assembly design should include}

(1). The structural strength related to its products must be considered in the design and must not affect its assembly. (2). The synchronous operation design is adopted to reduce the interference problem. 


\subsection{The green modular design that is easy to assemble should include}

(1). Synchronous operation design is adopted, and its ease of assembly is considered. (2). If the module design does not have a good guide slot, positioning is not recommended. (3). Attention should be paid to the combination of two objects and its positioning structure is involved. (4). The assembling items should have a foolproof structure design. (5). When designing the assembly items, pay attention to the reserved tolerances to reduce the efficiency of their assembly.

\subsection{For the unification of its parts, it must conform to the design of easy assembly, including}

Unifying the types of parts, standardizing the design, and unifying the parts.

\subsection{The disassembly of its parts conforms to the easy assembly design including}

(1). When disassembling one of its parts, it is no longer necessary to move unrelated parts. (2). Do not design structures that require special tools to disassemble.

\section{Conclusion}

This research process belongs to an empirical investigation method. From the research of a new model of a case company to its research results through a checklist, the actual vehicle design is used to improve its shortcomings. The research process and design findings are provided to design engineers A reference when designing. Therefore, after the physical design and green assembly and disassembly design review, the following review results can be obtained:

(1) Avoid future environmental pollution of parts that cannot be recycled.

(2) Increase the design review at each stage of the design to ensure that the required information is complete.

(3) The unification of parts and the reduction of the number and types of maintenance products.

(4) Confirm the possibility of materials, parts, easy assembly language and easy disassembly.

(5) Prevention of design changes and retests.

(6) Reduction of development cost and avoiding waste of energy.

\section{References}

1. Du Ruize, (1994) Research on Integrated System of Product Environmental Design, Journal of Daye, Volume III, First Issue, 103-116.

2. Gao Yulin (1991) Environmental protection leaderGerman packaging regulations and green dot twoway recycling system, product design and packaging, 48th issue, 66-69.
3. Guanneng Neng, Chen Yuande, (1993), Strategic research on applying green environmental protection concepts to product development, 1993 Industrial Design Technology and Proceedings of Academic Symposium, 11-19.

4. Wang Hongxiang, 1992, The Earth Speaks-Design Countermeasures for Environmentally Friendly Products, Product Design and Packaging, 50th Issue, 5-8.

5. Yuzhe Huang, (1999), Analysis and application of structural configuration of electric assisted bicycles at home and abroad, Bicycle Industry Research and Development Center.

6. Sakiguchi Shiro, (1990), Introduction to Design, Yifengtang Publishing House, P9-P15.

7. Xie Zhihe, (1997), Research on green assembly and disassembly design, taking notebook computer design as an example, Master's thesis of Institute of Industrial Design, Daye University.

8. Chen Mingxi, (1993), integrating the development of design and assembly auxiliary programs in the product design expert system, Master's thesis of Industrial Design Institute of Cheng Kung University.

9. Liu Yide, (1996.6), Research and analysis on the feasibility and trend of electric vehicle development, Master's thesis of Institute of Industrial Design, Daye University.

10. Lin Yingren, (1996), Research on the characteristics and evaluation of modular products from the perspective of structuralism, National Taiwan University Master's Thesis of Mechanical Engineering.

11. Hanchuan Lin (1995), Research on the conceptual design of modular structure, Master's thesis of Institute of Mechanical Engineering, National Taiwan University.

12. Yan Qinghui, (1995), Research on Computer Aided Disassembly Program Design and Evaluation Method of Green Design, Master's Thesis of Industrial Design Institute of Cheng Kung University

13. Shi Jiyuan, Dai Huashan, (2000), Product green design and application examples, Industrial Pollution Control No. 74, P177-P187.

14. Zeng Xiangwei, Xie Wenhe, (2000.3), Discussion on Design and Assembly Methods, CADESIGN P129-P130.

15. UCTURAL TESTING Part 1: Mechanical Mobility Measurements by Ole Dossing, Buruel and Kjar.

16. Ewin, D. J., (1984), Model Sting: Theory and Practice, Research Studies Press.

17. E.zussman, A. Kriwet, and G. Seliger, "disassembly-Oriented Assessment Methodology to Support Design for Recycling", Annals of the CIRP, Vol.43/1, pp .9-14,(1994)

18. Taipei Design Center Dusseldorf GMBHP product Assembly and Disassembly. (1994) 